This is the authors' pre-editorial version. Please refer to the original article as follows:

Hood, R, Gillespie, J. \& Davies, J. (2016) A conceptual review of interprofessional expertise in child safeguarding, Journal of Interprofessional Care, advance access, DOI:

10.3109/13561820.2016.1173656

http://www.tandfonline.com/doi/full/10.3109/13561820.2016.1173656 


\title{
A conceptual review of interprofessional expertise in child safeguarding
}

\begin{abstract}
It is increasingly accepted that practitioners across a range of professional fields must work together in order to promote children's welfare and protect them from harm. However, it has also become apparent that interprofessional working is a challenging area of practice that cannot simply be prescribed through protocols and procedures, nor acquired as a set of technical competences. This paper develops the concept of interprofessional expertise in order to explain how practitioners become more proficient at working with others to manage complex child welfare issues. Key principles are outlined with reference to relevant theoretical frameworks, including models of skill acquisition. The paper concludes by discussing some potential implications for future research and contemporary developments in child safeguarding practice.
\end{abstract}

\section{Introduction}

Most modern welfare states seek to improve the lives of children by providing support to vulnerable families, as well as intervening to protect children suffering abuse and neglect (Davies and Ward, 2012). The term 'safeguarding' therefore carries a dual sense of prevention and protection, with a balance of care and control functions (Own Author, 2015a). Safeguarding services can be visualised as a tiered structure, in which universal providers such as schools and general medical practice constitute a point of referral to more specialist services when additional needs are identified (Hardiker, 1991). For the children who are most at risk of harm, multi-agency interventions are coordinated by statutory 
protection agencies. Although mostly associated with social work, child safeguarding is actually an interprofessional area of practice. Greater complexity of need tends to imply more collaboration - for example, because multiple problems are unlikely to come within the remit of a single agency (Own Author, 2012). Equally, the risk of harm to children puts an onus on professionals to work together to make sure that signs of abuse are recognised and dealt with.

It will be argued here that a crucial part of effective safeguarding practice is interprofessional expertise, something that is hard to develop in mono-professional models of education and service delivery. The 'failure' of professionals to collaborate effectively, so often identified in child abuse tragedies, can be seen as the product of a system that retains expertise in professional silos (Own Author, 2015b). Procedural recommendations for professionals to hold meetings and share information can only accomplish so much in the absence of a sustained effort to educate practitioners and immerse them in the experience of joint working. However, before moving on to the concept of expertise, it is worth noting some important trends in interprofessional practice and education, as well as highlighting some jurisdictional differences.

\section{Trends in interprofessional practice and education}

While the need for interprofessional practice has become widely accepted, many countries have found their child safeguarding systems ill-equipped for it (Lonne \& Parton, 2014; Laming, 2004; Hughes, 2006). The reasons for this have been extensively discussed (Bunting, Lazenbatt, \& Wallace, 2010; Hawkins \& McCallum, 2001; Polnay, 2000; Raman, Holdgate \& Torrens, 2012). Services organised as separate professional bureaucracies inevitably create institutional and cultural barriers, which in child protection may be exacerbated by 
institutional anxiety about risk. For frontline practitioners, these issues are compounded by the ambiguity, unpredictability, and volatility of child welfare situations. The complexity of such cases can contribute to conflict and confusion in interprofessional networks, undermining an already difficult process of negotiation and coordination (Own Author, 2015c). It is therefore not surprising that there have been repeated calls for greater interprofessional learning across the relevant practice domains in health, education, and social care. Professionals working with children often find safeguarding to be an aspect of practice for which they feel poorly prepared, and the majority of professional groups continue to receive only basic child protection training (Goldman \& Grimbeek, 2011; Polnay, 2000; Rowse, 2009). Although the usual form of training continues to be monoprofessional, with a lack of opportunity for practitioners to learn 'with and from each other', there have been some efforts to integrate interprofessional education into child welfare programmes (Gilbert, Parton \& Skivenes, 2011).

In this respect, the UK has been noteworthy for the extent to which reforms to its child welfare system have emphasised collaboration and partnership. Since 2004, interorganisational structures called 'children's trusts' have contributed to the planning and provision of services in local areas, supplemented by safeguarding boards and other multiagency arrangements. Statutory guidance requires practitioners to form a 'team around the child' or 'core group' to implement child protection plans and meet the needs of the most vulnerable children (HM Government, 2015), while electronic workflow systems have attempted to embed a 'common language' for safeguarding practice (White, Hall \& Peckover, 2009). Some local authorities have experimented with new types of provision, with an emphasis on bringing practitioners together to work in integrated teams or 'social 
work units' (Goodman \& Trowler, 2012). A number of professions have introduced specialist training in child protection for both pre- and post-qualifying practitioners (Glennie, 2007), and courses drawing on interprofessional education have become increasingly common in higher education settings.

These changes have been significant, but have not been without their problems. Particularly in England, the emphasis on protocols and procedures, refracted through information technology, has been seen as contributing to excessive managerialism, blame culture and even 'magical thinking' in children's services (Wastell \& White, 2010). In practice, the tendency has been to coordinate the 'team around the child' as a series of specialist interventions rather than an integrated response to a complex situation (Own Author, 2012). A large body of research on 'barriers' and 'facilitators' to interprofessional working (see Brown \& White, 2006) has also allowed it to be construed as a technical problem, i.e. how to do more of the latter and less of the former. Recently, an influential review of child protection (Munro, 2011) explicitly advocated a 'socio-technical' approach, in which multiagency systems are set up to acknowledge complexity and manage it in a holistic fashion, rather than as an agglomeration of technical solutions to separate problems.

Developments in the UK and to some extent in other countries have therefore seen a concerted effort to bring professionals together to safeguard children, but also a struggle to resolve the additional problems incurred by doing so. It cannot be assumed that the ability to collaborate with others is acquired as a natural corollary of becoming a professional. Indeed, much of the research cited above seems to suggest the opposite, i.e. working across boundaries, roles and remits can be difficult for practitioners precisely because they - and their agencies - have been encouraged to specialise (Gilbert et al., 2011). The question then 
arises as to what constitutes the expertise to work interprofessionally, and how it is constituted.

\section{The concept of expertise}

Bradley, Paul \& Seeman (2006: 77) describe an expert as 'someone who is characterised by superior performance within a specific domain of activity'. Accordingly, expertise consists of the skills, knowledge and practices that enable professionals to develop such proficiency, and whose emergence is influenced by political, educational and organisational contexts. The degree to which a practitioner is considered an expert will depend as much on their individual abilities and experience as on their formal qualification. In much of the literature, expertise is discussed in relation to a set of dualities including:

- Progression over time from novice to expert (Benner, 1984; Dreyfus \& Dreyfus, 1986)

- Development of formal/propositional knowledge and non-formal/tacit knowledge (Kinchin \& Cabot, 2010; Eraut, 2000)

- Application to problems characterised by complexity and uncertainty, and which are not amenable to technical solutions (Fook, Ryan \& Hawkins, 2000; Rittel \& Webber, 1973)

Table 1 considers these dualities in further detail, in order to reveal some of the key assumptions underlying the concept of expertise.

\begin{tabular}{|l|l|}
\hline Novice & Expert
\end{tabular}

- Adheres rigidly to rules and protocols

- Relies on intuitive grasp of situations 


\begin{tabular}{|c|c|}
\hline $\begin{array}{l}\text { - Perceives only individual aspects of } \\
\text { situation } \\
\text { - Relies on analytical approaches } \\
\text { - Reluctant to use discretion }\end{array}$ & $\begin{array}{l}\text { - Perceives situations holistically, sees } \\
\text { underlying issues } \\
\text { - Selectively uses analytic approaches } \\
\text { - Creative and confident in finding solutions }\end{array}$ \\
\hline $\begin{array}{l}\text { Formal knowledge } \\
\text { - Can be codified as propositions, rules } \\
\text { - Acquired in a formal process of learning } \\
\text { - Involved in analytic mode of cognition }\end{array}$ & $\begin{array}{l}\text { Tacit knowledge } \\
\text { - Cannot be codified and is often difficult to } \\
\text { - Acquired through (reflection on) practice } \\
\text { - } \text { Involved in intuitive mode of cognition } \\
\text { - Relies on individual skills and abilities } \\
\text { - Personal and context-dependent (so often } \\
\text { lost during employee turnover) }\end{array}$ \\
\hline $\begin{array}{l}\text { Technical problems } \\
\text { - Can be formulated with all the } \\
\text { necessary information needed to find a } \\
\text { - Have a clear measure of success and an } \\
\text { - Solution should apply to all similar } \\
\text { problems }\end{array}$ & $\begin{array}{l}\text { Complex problems } \\
\text { - Have no definitive formulation } \\
\text { - Relate to multiple issues so may be difficult } \\
\text { recognise when an end-point has been } \\
\text { - Have a unique configuration, so a 'solution' } \\
\text { may not work in other cases }\end{array}$ \\
\hline
\end{tabular}




\section{Table 1. Dualities and assumptions underlying expertise}

The 'skill acquisition model' of expertise has been studied in many professions, including teaching (Berliner, 1994), nursing (Benner, 1984), and social work (Fook et al., 2000), but has also come in for some criticism. In particular, the model is associated with a privileging of tacit knowledge that may downplay the importance of analytical processes based on formal learning (Norman, 2005), or unnecessarily construct novices as observers who follow rules (Dall'Alba \& Sandberg, 2006). The association of expert practice with 'unknowable' mental processes of intuition and tacit understanding is by definition hard to prove, and other explanations seem equally valid (e.g. Schön, 1991). More recent studies suggest that expertise requires well-organized structures of both formal and tacit knowledge, and that experts combine these two forms of knowledge in a dual process that creates tailored connections in order to solve complex problems (Kinchin \& Cabot, 2010). Although expertise is often associated with rapid assessment and response, experts may spend more time than novices determining an appropriate representation of and response to the problem at hand, in part because they have more sophisticated repertoires of dual knowledge processing (Bradley et al., 2006). While the nature of tacit knowledge makes it challenging to articulate outside of the context, there are arguments that it can be accessed through reflection and discussion regarding what one is doing and why within a specific situational context (Luntley, 2011).

Other studies have emphasised the social dynamics that nurture professional identity and behaviour in work settings. For example, Wenger (1998) describes how learning occurs through collective participation in what he calls 'communities of practice'. Over time, 
through a cumulative process of dialogue and shared experience, practitioners develop a repertoire of resources, including stories, experiences, tools, and techniques, which help them to interpret and solve problems. A complementary perspective is supplied by activity theory (e.g. Engestrom, 1999), which analyses how different elements (people, tools, rules, divisions of labour) coalesce around the 'object' of the activity system. The result is a nexus of different perspectives, practices and expertise that is characterised by change and innovation, rather than stability and consensus. In the field of education research, for example, Daniels, Edwards, Engeström, Gallagher, \& Ludvigsen (2013) used activity theory to illustrate the provision of services through emergent networks of agencies, professionals and clients, rather than through monolithic organisational structures. Expertise in such settings is distributed across services and agencies, with collaboration having to be improvised in focused, time-limited bursts of activity around particular cases.

The literature considered here offers a professional understanding of expertise, focused on how informal and formal networks of knowledge develop over time, and in action, within a specific domain. Admittedly, there are other approaches to expertise. A sociological perspective, for example, might focus on the claims to specialist skills and knowledge made by occupational groups as part of their 'professional project' (Larson, 1977), or look at how different discourses of expertise are deployed within interprofessional settings (Own Author, 2014). Issues of status and hierarchy have been found to be important factors in collaborative contexts (e.g. Brown and White., 2006), while the perception of who is and is not an (or the) 'expert' in a child protection case may be contested on different levels, given professional and institutional anxieties about risk and responsibility (Own Author, 2015b). The argument made here is that all of these factors are part of what makes interprofessional 
working a complex area of practice in its own right. Practitioners will therefore develop a characteristic blend of formal and experiential knowledge in relation to interprofessional practice, a form of expertise that borrows from but is distinct to expert practice in their own specialist fields.

\section{Developing interprofessional expertise}

Given the possibilities and limitations explored above, what are the implications of seeing proficiency in working with others as a form of expertise? To begin with, we must accept that professionals are not necessarily good at working with each other just because they are experts in their own field. Indeed, as noted earlier, the opposite might sometimes be true. The progression from novice to expert is associated with experience but again it cannot be assumed that professionals will learn simply as a result of being 'thrown together' in meetings, case conferences, and so on. That is not how one trains professionals, after all. Instead, interprofessional expertise will be acquired by practitioners with the cognitive ability to understand and apply different kinds of knowledge in working with others to resolve complex situations. The concept is illustrated below in Figure 1.

Figure 1. Interprofessional expertise in child safeguarding

Figure 1 suggests a domain-specific application of general attributes of collaborative practice, which may be acquired and demonstrated by a range of professionals involved in child safeguarding. We can see that a number of contextual variables are involved. Legal and institutional frameworks, including safeguarding procedures, set out the arrangements through which collaboration occurs. Education and training is necessary to establish a common grounding in safeguarding principles, recognise different professional roles and 
perspectives, and gain awareness of what skills are needed to work effectively with others. This knowledge is then applied in the context of working interprofessionally on complex safeguarding cases. In the process, practitioners should attain some of the competences outlined by Barr (1998: 185), such as the ability to 'describe one's roles and responsibilities clearly to other professions and discharge them to the satisfaction of those others', or to 'recognise and respect the roles, responsibilities and competence of other professions in relation to one's own, knowing when, where and how to involve those others through agreed channels'. In time, they will also develop the 'intuitive grasp of situations based on deep tacit understanding' (Eraut, 2000: 126) that allows them to deal with the unique challenges and dynamics of collaboration in each particular case.

Similar ideas are discussed by Charles, Bainbridge \& Gilbert (2010), who suggest that timing is a critical component in developing new perspectives on professional interaction. In their model of interprofessional education, practitioners go through three overlapping stages: exposure, immersion, and mastery. Exposure is intended to occur during pre-qualifying education, while students are developing in depth knowledge of their own chosen profession; the goal is for students to add to this knowledge a preliminary appreciation of the existence of different world views and the roles of other professions. Immersion is intended to occur once students or new professionals have a firm grasp on the knowledge base and roles of their own profession and have had exposure to other professionals in action; the desired outcome of this stage is an 'interprofessional world view' (2010: 15) that recognizes and values the roles and contributions of others. Mastery, the final stage, consists of advanced level critical thinking skills, along with the tacit knowledge to fully 
contribute on interprofessional teams and to articulate collaborative concepts and skills to others.

The concept of expertise therefore emphasises the extent to which encouraging 'interprofessionalism' - as opposed to silo-based professionalism - depends on providing practitioners with the opportunity to learn and work together in a supportive context. As noted above, the contextual variables can vary quite significantly between agencies, professions and jurisdictions, e.g. statutory roles and responsibilities, the significance awarded to training on child welfare, and to collaboration with other professionals. Members of 'core groups' in child protection cases will have divergent degrees of professional involvement ranging from a half a day a month to several days a week (Hallet \& Stevenson, 1980; Willumsen, 2008). Moreover, such groups are rarely part of co-located teams under unitary management but tend to be combined in loosely structured networks, across which expertise is 'distributed' (Daniels et al., 2013). Clearly these factors will lead not only to differences in formal knowledge but also the kind of tacit knowledge that is central to the development of expertise.

Of course, the elicitation and articulation of tacit knowledge is one of the bugbears of research into expertise. In principle it should be possible to distinguish between 'expert' practice and practice which is merely 'competent' because of a learned proficiency in carrying out routine activities (Collins, 2004; Fook et al, 2000). To explore what this distinction might look like in practice, it is worth considering one of the staples of safeguarding practice: the multi-agency meeting. In most jurisdictions, there will be a requirement for services to convene such a meeting when there are concerns that a child may be at risk of abuse or neglect. If the concerns seem to be substantiated, a multi-agency 
protection plan is drawn up and a series of further meetings are held to review progress. For professionals involved in this process, there are various layers to the collaborative activity associated with these meetings, some of which are outlined below in the form of reflective questions:

- Procedure - how often do these meetings happen, where are they being held, do I have to attend; do I have to provide a report?

- Role and remit - what are my responsibilities, what information do I need to share, what are the roles of other professionals?

- Knowledge - what do I know about the signs and effects of abuse, what do I know about this child, what do I know about the parents and family, what do I know that other professionals don't (and vice versa)?

- Assessment - how worried am I about this child, what has happened to make things worse, what are the strengths of the family, how would I know if things were getting better?

- Risk - what is the worst that could happen, how likely is that, would I be blamed, what if I'm wrong?

- Intervention - what needs to happen before I'm confident this child will be OK, what can I do, what do other professionals need to do, how soon does this need to happen?

- Relationship with family - what do I say to the parents, how will they react, how can I maintain the relationship?

- Relationship with professionals - who do I know, what do they think, do we agree with each other, are we all trusted equally by the family? 
- Conduct of meeting - who is in charge, do I explain to the parents about the meeting or should someone else do that, do I speak to other professionals beforehand, how do I decide what to say and how to say it, will my voice be heard, what if I disagree with someone?

It is not suggested that every professional reflects on all of these issues before every meeting. Indeed, that is part of the point. Nonetheless, it should be clear from this far-fromexhaustive list that there is plenty of scope for practitioners to develop their thinking about working with others as they gain more experience. The progression towards expertise may even be evident in how openly and constructively such questions are explored. For example, instead of concentrating on their own professional involvement relative to that of others, the interprofessional practitioner may wish to consider how the group can shape a collective approach in partnership with the family (Own Author, 2015c). 'Efficient, open and equitable communication' (Hewitt, Sims \& Harris., 2014) can be deployed not only to share information and clarify one's role to others, but also to acknowledge and harness disagreements in search of creative solutions. Meetings are not the sole forum for interprofessional working but form part of an ongoing process of dialogue, discussion and debate; their format does not have to be pre-determined but can be designed for making decisions, setting goals and reviewing plans in specific circumstances. None of these things happen automatically, and demand more than just complying with a protocol initiated by the statutory social worker. As such, the conditions for successful collaboration emerge from the particular and will vary from case to case.

\section{Discussion and implications for research}


It has been argued that working interprofessionally to safeguard children's welfare constitutes a domain of expert practice, which encompasses elements of formal and tacit knowledge, depends on the ability and experience of the practitioner, and is shaped by their role as well as the jurisdiction in which they have trained and worked. The significance of such expertise lies in its contribution to effective collaboration in complex cases, which demand more than routine coordination and knowledge of procedures. On this basis it can be hypothesised that differences in interprofessional expertise will be evident in children's practitioners across services and jurisdictions, and between those who discharge different roles and remits in relation to safeguarding. Indeed, interprofessional expertise could be considered one of the mechanisms of collaboration and teamwork, which enable practitioners to work across boundaries, share responsibility and critically review their collective decisions (Hewitt et al., 2014). What is needed is more empirical work to explore elements of this type of expertise and how they emerge in different contexts and at different stages of professional development.

The findings should be of interest to frontline services as well as higher education settings. The prevalent model of monoprofessional training, with its creation of distinct professional cultures and identities, may constitute a barrier to effective collaboration once people are out in practice (Hall, 2000). If safeguarding is increasingly being conceived and delivered as an multi-agency service, as developments in the UK and elsewhere seem to suggest, this presents a challenge to the structure of pre- and post-qualifying programmes, especially for practitioners destined to specialise in children and families work. However, similar considerations apply in other areas of health and social care, in which services now have to manage growing numbers of people with a cluster of chronic conditions, including physical 
and mental multimorbidity (Mercer et al., 2012). Considering this challenge in terms of interprofessional expertise highlights the need for practitioners at various stages to learn and work together, and for placements and practicums to incorporate this as an essential rather than supplementary form of experience. At the same time, it should be recognised that skill acquisition is not just a matter of intuitive understanding and practice wisdom, but also relies on formal knowledge and a critical understanding of research evidence (Munro, 2008).

For service providers, the topic raises a number of questions. For co-located multi-agency teams such as family recovery projects (Thoburn, 2015) or multi-agency safeguarding hubs (Crockett et al., 2013), it would seem important to know how to identify and develop interprofessional expertise, e.g. in the recruitment and training of staff. With respect to standard safeguarding processes, which require the coordination of distributed networks such as the 'team around the child', other issues arise. For example, professions such as nursing and teaching have tended to manage these processes through clinical leads and consultant roles within agencies (Abbott, 2007). Such roles might be expected to foster interprofessional expertise in the people performing them, who would have the opportunity to develop specialist knowledge of safeguarding as well as experience of collaborating with others. However, the corollary of this might be an overreliance on those lead practitioners by their colleagues, and a corresponding loss of expertise when they were absent or left their role. As with all organisational structures, the challenge is not to unwittingly create silos, which make boundary crossing difficult and induce anxiety in workers faced with issues they perceive as belonging elsewhere (Cilliers and Greyvenstein, 2012). 


\section{Conclusion}

This paper has drawn on a range of literature to build a conceptual framework for interprofessional expertise, aiming to explore how practitioners might develop collaborative competences but also an experiential understanding of joint working in particular cases and contexts. Given the diverse approaches to safeguarding among different professions and states, it would seem important to conduct cross-jurisdictional work in this area. The literature on child welfare has long recognized the value of comparative studies and has shown how the policy context shapes attitudes to collaboration as well as its institutional forms. Comparisons across professional groups and team typologies might also shed some insight into which factors were most influential in developing competence and understanding of joint working within 'common' frameworks of assessment and intervention. As always, there remains plenty of scope to develop our knowledge of this important area of interprofessional practice. 


\section{References}

Abbott, S. (2007). Leadership across boundaries: a qualitative study of the nurse consultant role in English primary care. Journal of Nursing Management, 15(7), 703-710.

Barr, H. (1998). Competent to collaborate: Towards a competency-based model for interprofessional education. Journal of Interprofessional Care, 12(2), 181-186.

Berliner, D. C. (1994). Expertise: The wonders of exemplary performance. In John N. Mangieri and Cathy Collins (Eds.) Creating powerful thinking in teachers and students (pp. 141-186). Ft. Worth, TX: Holt, Rinehart and Winston.

Bradley, J. H., Paul, R., \& Seeman, E. (2006). Analyzing the structure of expert knowledge. Information \& management, 43(1), 77-91.

Brenner (1984). From Novice to Expert. New York: Addison Wesley

Briar-Lawson, K., \& Zlotnik, J. L. (2014). Charting the impacts of university-child welfare collaboration. New York: Routledge.

Brown, K. \& White, K. (2006). Exploring the evidence base for Integrated Childrens Services. Retrieved from http://www.openscotland.gov.uk/publications

Bunting, L., Lazenbatt, A. \& Wallace, I. (2010). Information sharing and reporting systems in the UK and Ireland: Professional barriers to reporting child maltreatment concerns. Child Abuse Review, 19(3), 187-202.

Charles, G., Bainbridge, L., \& Gilbert, J. (2010). The University of British Columbia model of interprofessional education. Journal of Interprofessional Care, 24(1), 9-18. 
Cilliers, F., \& Greyvenstein, H. (2012). The impact of silo mentality on team identity: An organisational case study. SA Journal of Industrial Psychology, 38(2), 75-84.

Collins, H. (2004). Interactional expertise as a third kind of knowledge. Phenomenology and the Cognitive Sciences, 3(2), 125-143.

Crockett, R., Gilchrist, G., Davies, J., Henshall, A., Hoggart, L., Chandler, V., \& Webb, J. (2013). Assessing the early impact of Multi Agency Safeguarding Hubs (MASH) in London. London: London Councils and University of Greenwich.

Daniels, H., Edwards, A., Engeström, Y., Gallagher, T., \& Ludvigsen, S. R. (Eds.). (2013). Activity theory in practice: Promoting learning across boundaries and agencies. Abingdon: Routledge.

DallAlba, G. \& J. Sandberg. (2006). Unveiling professional development: A critical view of stage models. Review of Educational Research, 76, 383-412.

Dreyfus, H. \& Dreyfus, S. (1986). Mind over machine: The power of human intuition and expertise in the era of the computer. New York: The Free Press.

Engeström, Y. (ed.) (1999) Perspectives on activity theory. New York: Cambridge University Press.

Eraut, M. (2000). Non-formal learning and tacit knowledge in professional work.British Journal of Educational Psychology, 70(1), 113-136.

Fook, J., Ryan, M. \& Hawkins, L. (2000). Professional expertise: Practice, theory and education for working in uncertainty. London: Whiting \& Birch. 
Gilbert, N., Parton, N., \& Skivenes, M. (Eds.). (2011). Child protection systems: International trends and orientations. Oxford: Oxford University Press.

Glennie, S. (2007). Developing interprofessional relationships: Tapping the potential of interagency training. Child Abuse Review, 16(3), 171-183.

Goldman, J. \& Grimbeek, P. (2014). Bridging the gaps in child sexual abuse reporting and disclosure: Child sexual abuse and mandatory reporting intervention preservice content preferred by student teachers. Journal of Child Sexual Abuse, 23, 1-16

Goodman, S. \& Trowler, I. (eds.) (2012) Social Work Reclaimed. London, Jessica Kingsley. Hall, P. (2005). Interprofessional teamwork: Professional cultures as barriers. Journal of Interprofessional care, 19(S1), 188-196.

Hallett, C. \& Stevenson, O. (1980). Child Abuse: Aspects of Professional Cooperation. Allen and Unwin: London

Hardiker, P., Exton, K. \& Barker, M. (1991). Policies and Practices in Preventive Child Care. Ashgate: Aldershot.

Hawkins, R., \& McCallum, C. (2001). Effects of mandatory notification training on the tendency to report hypothetical cases of child abuse and neglect. Child Abuse Review, 10(5), 301-322.

Hewitt, G., Sims, S., \& Harris, R. (2014). Using realist synthesis to understand the mechanisms of interprofessional teamwork in health and social care. Journal of interprofessional care, 28(6), 501-506. 
HM Government (2015) Working together to safeguard children: A guide to inter-agency working to safeguard and promote the welfare of children. London: The Stationery Office Hughes, E. N. (2006). BC children and youth review: An independent review of British Colombia's child protection system. Victoria, BC: Ministry of Children and Family Development.

Kinchin, I. \& Cabot, L. (2010). Reconsidering the dimensions of expertise: from linear stages towards dual processing. London Review of Education, 8(2), 153-166.

Larson, M. S. (1977). The Rise of Professionalism: A Sociological Analysis. University of California Press: London Lonne, B., \& Parton, N. (2014). Portrayals of child abuse scandals in the media in Australia and England: Impacts on practice, policy, and systems. Child abuse \& neglect, 38(5), 822836.

Luntley, M. (2011). What do nurses know? Nursing Philosophy, 12(1), 22-33.

Mercer, S. W., Gunn, J., Bower, P., Wyke, S., \& Guthrie, B. (2012). Managing patients with mental and physical multimorbidity. British Medical Journal, 345: e5559

Munro, E. (2008). Effective Child Protection. Sage: London.

Munro, E. (2011) The Munro Review of Child Protection: Final Report. A child-centred system. London, The Stationery Office.

Norman, G. (2005). Research in clinical reasoning: past history and current trends. Medical education, 39(4), 418-427. 
Polnay, J. C. (2000). General practitioners and child protection case conference participation: reasons for non-attendance and proposals for a way forward. Child Abuse Review, 9(2), 108-123.

Raman, S., Holdgate, A., \& Torrens, R. (2012). Are Our Frontline Clinicians Equipped with the Ability and Confidence to Address Child Abuse and Neglect? Child Abuse Review, 21(2), 114130.

Rittel, H. \& Webber, M. (1973). Dilemmas in a General Theory of Planning . Policy Sciences, 4, 155-169.

Rowse, V. (2009). Childrens nurses' experiences of child protection: what helps? Child Abuse Review, 18(3), 168-180.

Schön, D. A. (1991). The Reflective Practitioner: How Professionals Think in Action. Aldershot: Avebury Academic Publishing.

Suter, E., Arndt, J., Arthur, N., Parboosingh, J., Taylor, E., \& Deutschlander, S. (2009). Role understanding and effective communication as core competencies for collaborative practice. Journal of interprofessional care, 23(1), 41-51.

Thoburn, J. (2015) The Family Recovery Approach to Helping Struggling Families, in Davies, K. (ed.) Social Work with Troubled Families: A Critical Introduction. London: Jessica Kingsley. White, S., Hall, C. \& Peckover, S. (2009). The Descriptive Tyranny of the Common Assessment Framework: Technologies of Categorization and Professional Practice in Child Welfare. British Journal of Social Work, 39(7), 1197-1217. 
Wastell, D. \& White, S. (2010). Technology as magic: fetish and folly in the IT-enabled reform of childrens services. In Ayre, P. \& Preston-Shoot, M. (Eds.), Childrens Services at the Crossroads: A Critical Evaluation of Contemporary Policy for Practice. Lyme Regis: Russell House Publishing.

Wenger, E. (1998). Communities of Practice. Cambridge University Press: Cambridge.

Willumsen, E. (2008). Interprofessional collaboration - a matter of differentiation and integration: Theoretical reflections based in the context of Norwegian childcare. Journal of Interprofessional Care, 22(4), 352-363. 